\title{
Remote Sensing Information Processing Grid Node with Loose-Coupling Parallel Structure
}

\author{
Ying Luo ${ }^{1,3}$, Yong Xue ${ }^{1,2, *}$, Yincui $\mathrm{Hu}^{1}$, Chaolin $\mathrm{Wu}^{1,3}$, Guoyin Cai ${ }^{1}$, Lei Zheng ${ }^{1,3}$, \\ Jianping Guo ${ }^{1,3}$, Wei Wan ${ }^{1,3}$, and Shaobo Zhong ${ }^{1}$ \\ ${ }^{1}$ State Key Laboratory of Remote Sensing Science, Jointly Sponsored by the Institute of \\ Remote Sensing Applications of Chinese Academy of Sciences and Beijing Normal University, \\ Institute of Remote Sensing Applications, Chinese Academy of Sciences, P.O. Box 9718, \\ Beijing 100101, China \\ ${ }^{2}$ Department of Computing, London Metropolitan University, 166-220 Holloway Road, \\ London N7 8DB, UK \\ ${ }^{3}$ Graduate School of the Chinese Academy of Sciences, Beijing, China \\ jennyjordan@hotmail.com, y.xue@londonmet.ac.uk
}

\begin{abstract}
To use traditional algorithms and software packages on Grid system, traditional algorithms and software packages, in general, have to be modified. In this paper we focus on standards and methodologies for Grid platform within the context of the Remote Sensing Data Processing Grid Node (RSDPGN) that implements a loose-coupling parallel structure for orchestrating traditional remote sensing algorithms and software packages on the Condor platform. We have implemented 17 remote sensing applications in one system using Web service and workflow technology without any change to traditional codes. Some core algorithm codes are come from a remote sensing software package which we has neither resource codes nor APIs. Others come from the program codes accumulated by our group. The design and prototype implementation of RSDPGN are presented. The advantage and shortage of loose-coupling structure is analysed. Through a case study of land surface temperature calculation from MODIS data, we demonstrate the way to modify software packages in details. Moreover we discuss the problems and solutions based on our experience such as system architecture, the kinds of functional modules, fast data transfer, and state monitoring.
\end{abstract}

\section{Introduction}

The information extracted from remote sensing data plays an important role in science and society. The algorithms and software packages to extract information vary based the characteristics of sensors, spectrum, etc. Generally speaking, the process of remote sensing data is complicated and time consuming. Remote sensing software packages such as ARCGIS, ENVI, and ERDAS are expensive, and the use of them needs special skill. On the other hand, researchers might prefer to pay for only the function modules which he or she is interested in, or have a third party to do the preprocessing steps or offer some computing power on demand. Thus we need a system

\footnotetext{
${ }^{*}$ Corresponding author.
} 
that provides individual function and computing power, allow user to pay for only certain functions. The emergence of Grid technology brings out a solution to this system. Grid technology is an evolution in resources sharing including hardware, data, and software. It will change the mode of software industry at both develop stage and sale stage.

The paper is organized as follows. In section 2 RSDPGN with some detail of the functions of the various components and our implementation at present will be demonstrated. Then in section 3, we will discuss the standards and methodologies for Grid platform through a case study of land surface temperature retrieval from MODIS data, and give our experiences in building RSDPGN on how to build a computing Grid node. Finally, the conclusions will be addressed in Section 4.

\section{Remote Sensing Data Processing Grid Node (RSDPGN)}

Remote Sensing Data Processing Grid Node (RSDPGN) is the infrastructure that manages the software and hardware resources to process remote sensing data according to users' demand. The data to be processed by our node is come from the user or a third party.

\subsection{Architecture of RSDPGN}

Figure 1 describes the architecture of remote sensing data processing Grid node. The node has four layers:

1) Portal layer: It is an outmost user interface, which can display data and results, receive data, $\mathrm{JOB}$, and algorithm, register and certificate.

2) Application layer: It is the most important layer for a processing node. The fundamental modules include reliance file transfer (RFT), security, several database (DB) such as algorithm database, remote sensing data database, user infor-

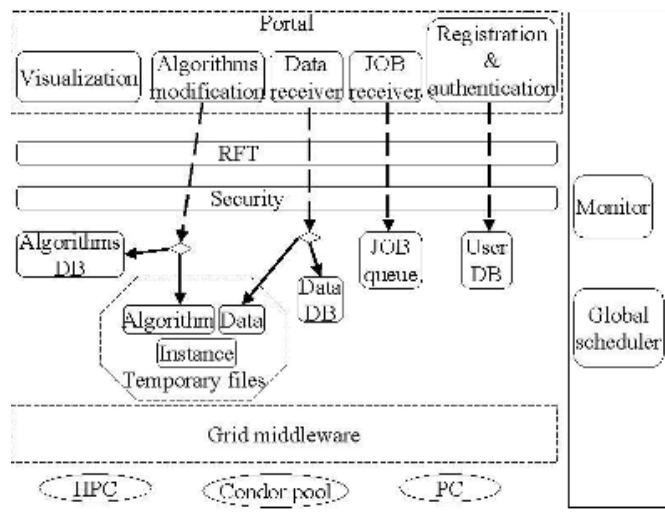

Fig. 1. Architecture of RSDPGN mation database, and instance information database, monitor to check the status of JOB, hardware, and software, and global scheduler.

3) Grid middleware layer: The communication and management to hardware resource is in this layer. We developed a toolkit named RSNkit (Remote Sensing Node toolkit) to add some functions not provided by Condor.

4) Hardware layer: The hardware entities can be PCs, HPC, or Condor pools, etc.

The node issues and registers its services to SIG manage centre periodically, responses calls of SIG, triggers services, and reports status. There an instance of an 
application is called a JOB. A JOB has several steps. Each step is one task that can be submitted to either the Condor pool or HPC or PC. Receiving require from SIG manager, the node will find data from user or remote data servers according to user's requirements, organize computing resource dynamically, trigger services, and monitor their running status. To decrease the total processing time of a task, the node will divide a task into several sub-tasks. The exact number and size of the sub-tasks is according to the current PC number and configure in the Condor computing pool [1] and super computer or designated by the user. Only when the task is large enough, or on the user's request, the node triggers off the super computer to do a large sub-task. The method we trigger super computer is different with that of Condor pool.

\subsection{Implementation of RSDPGN}

Currently, RSDPGN provides 17 remote sensing applications by MODIS data, such as aerosol optical depth retrieval, land surface temperature retrieval, soil moisture retrieval, surface reflectance retrieval, some vegetation indexes, and a series of preprocessing functions. Some core algorithm codes of remote sensing applications are from a remote sensing software package, which we has neither resource codes nor APIs. Others are program codes accumulated by our group. We implement our portal as a Web site using dynamic page technology JSP. The development work of end user software is minimum. Anyone who has a browser can use our node. In order to integrate with other Grid systems in the future, our node is implemented with Web service technology such as SOAP, XML, and WSDL. Each remote sensing application is enwrapped individually so that the system is loose coupling and parallel structure. There's no global control as normal system. Each remote sensing application manages itself following the pre-defined strategy in workflow. In other words, the whole system is not a large workflow, but many small parallel workflows classified by applications. Only some basic function modules such as monitor, JOB queue, RFT are used.

\section{Case Study}

In this research, we will use an example of land surface temperature retrieval from MODIS data to demonstrate how to use our node [2]. The retrieval process consists of initialisation, data format transfer, rectification, region selection, data division, temperature retrieval, result merging and result return to the users. When a user orders a temperature retrieval service via Grid portal, a JOB workflow instance is initialised once the user click the submit bottom after choosing the data to be processed. Finally the blocks of retrial result are merged, and return to the end user. The JOB status is monitored by the system monitor. The monitor of the whole node including JOB status, sub jobs' status, workflow module status, hardware status is also an important issue. Continually status query wastes much CPU cycles. Our solution is to give a speed attribute to each algorithm. So the system can estimate a probable time and use the time to decide whether to query the status.

File transfer is one of the big issues. It is the bottleneck that increases the total processing time. There is another issue adding the complexity of the data node. The 
application workflow need to know not only data and metadata but also attributes such as whether it has been rectified, with what algorithm, how about the processing precision, and so on.

\section{Conclusion}

In this paper, we introduced our ongoing research on RSDPGN. It is a demonstration on how to integrate traditional algorithms and software packages, computing resource, and Grid in order to provide one-stop service to users. It is service-oriented. We have implemented it mainly by workflow technology. We orchestrated 17 remote sensing applications on the Grid node. We used the strategy to let each application manages itself following the pre-defined workflow. The loosely coupling structure of our node is different with common centralized control structure. It is an easy design, modifying, and extending system. Furthermore this structure is convenient to breakpoint and status query, but it adds spending of computing resource and store resource. Additionally, the rock-bottom communication, control and monitor to Condor computing pool and HPC rely on Condor and RSNkit. It is recommended to use loosely coupling structure in complex Grid system that involved in many function modules and hardware. But for the correspondingly system, it is better to use a centralized control structure.

\section{Acknowledgement}

This publication is an output from the research projects "Grid platform based aerosol fast monitoring modeling using MODIS data and middlewares development" (40471091) funded by the NSFC, China, "Dynamic Monitoring of Beijing Olympic Environment Using Remote Sensing" (2002BA904B07-2) and "863 - Remote Sensing Information Processing and Service Node" (2003AA11135110) funded by the MOST, China and "Digital Earth" (KZCX2-312) funded by CAS, China.

\section{References}

[1] Basney, J., Livny, M., and Tannenbaum, T., 1997, High Throughput Computing with Condor, HPCU news, Volume 1(2)

[2] Xue, Y., Cai, G. Y., Guan, Y. N., Cracknell, A. P. and Tang, J. K., 2005, Iterative SelfConsistent Approach For Earth Surface Temperature Determination. International Journal of Remote Sensing, Vol. 26, No. 1, 185-192. 